\title{
Orbital Lymphagioma: About A Case Report
}

\author{
H. EL HAMMAOUI ${ }^{*}$, H EL MORTAJI ${ }^{1}$, M. EL GHAIDI ${ }^{1}$, D BASRAOUI ${ }^{1}$, H.Jalal ${ }^{1}$, R BOUDA ${ }^{2}$, H ABOU EL
} HOUDA $^{2}$, Y EL GHANI $^{2}$, S BELGHMAIDI $^{2}$, I HAJJI ${ }^{2}$, A MOUTAOUAKIL $^{2}$

\footnotetext{
${ }^{1}$ Radiology Department, Mother and Child Hospital, CHU Med VI, Marrakech

${ }^{1}$ Ophtalmology Department, CHU Med VI, Marrakech
}

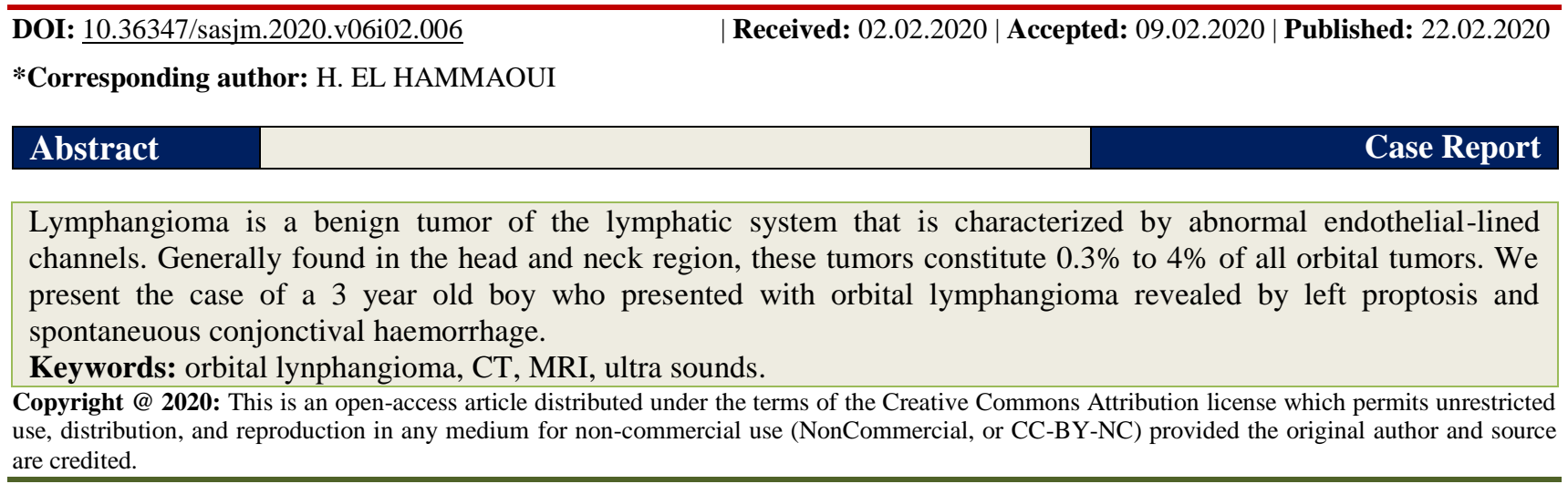

\section{INTRODUCTION}

Lymphangiomas are benign hamartomatous tumours usually diagnosed in early childhood. About $20 \%$ of these tumours involve the orbit and ocular adnexa; they account for less than $2 \%$ of orbital biopsies [1].

Clinically, lymphangiomas usually present with a slowly progressive proptosis, displacement of the globe, ptosis, and restriction of eye movements; rarely focal lesions may remain asymptomatic. Spontaneous intraorbital haemorrhage may cause acute proptosis, compressive optic neuropathy, and loss of the vision. In children who developed marked ptosis deprivation amblyopia may develop [2]. We present the case of a left orbital lymphangioma in a 3 year old child.

\section{CASE REPORT}

A 3-year-old boy was admitted in our radiology department with two weeks-history of left proptosis and spontaneous conjonctival haemorrhage of the left eye (figure 1).

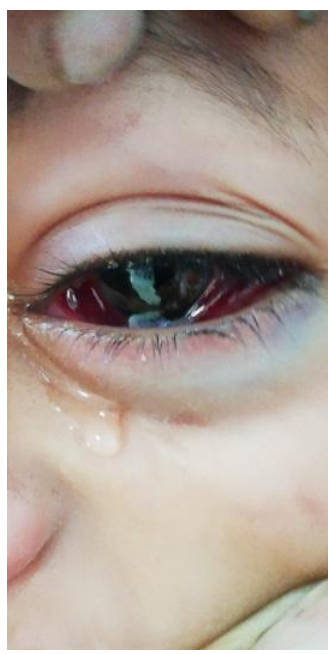

Fig-1: Spontaneous conjonctival haemorrage of the left eye

A CT showed a lobulated intraconal masse in the left orbit. It was hypodense with no significant enhancement (figure 2). 


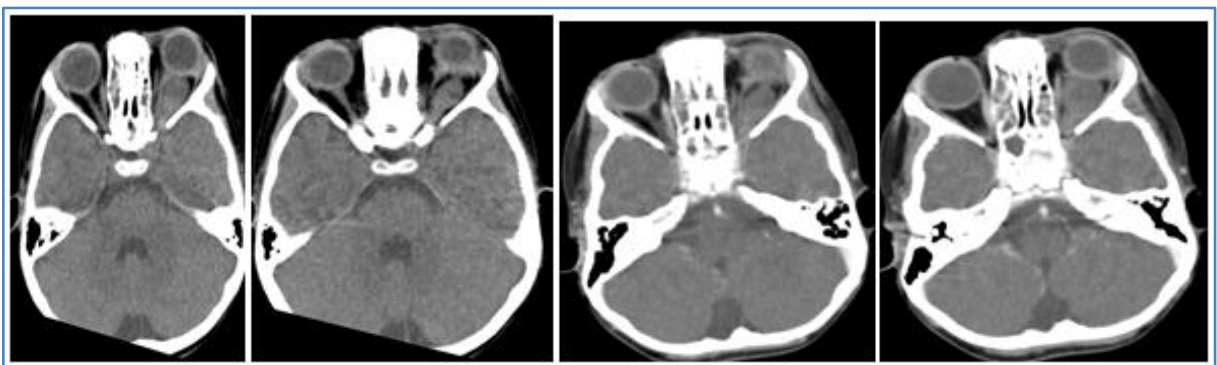

Fig-2: CT with and without contrast: lobulated intraconal masse in the left orbit; hypodense with no significant enhancement

MRI was performed for better characterization and showed an unencapsulated and lobulated intraconal cystic masse in the left orbit. It was deeply located behind the ocular glob. The septated cytic mass was heterogenious in T1 weighted view with a hyposignal and hypersignal content correponding to intracystic haemorrhage. The T2 weighted images showed a hypersignal with a fluid level within the mass. The mass also showed enhancement of its internal septation following contrast administration and was responsible of a compression on of the optic nerve (figure 3 ). An orbital lymphangiom was evoqued.
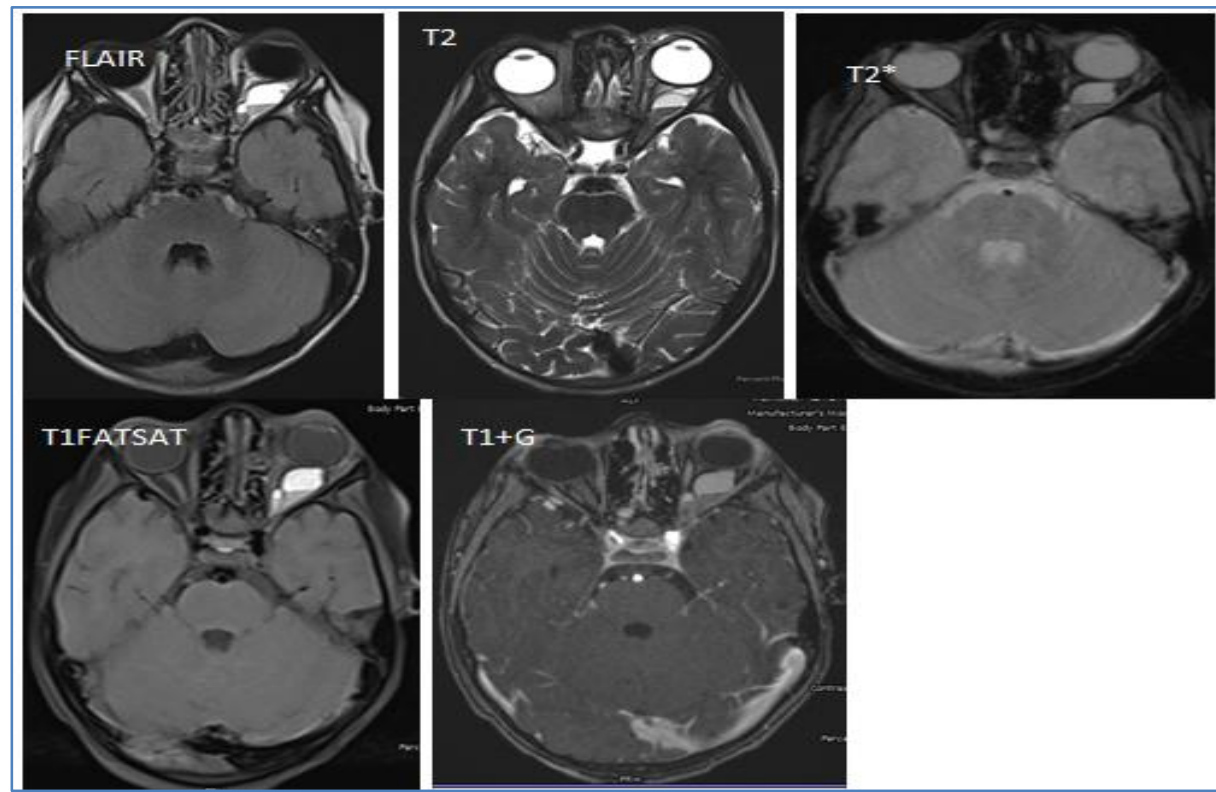

Fig-3: Unencapsulated and lobulated intraconal cystic masse in the left orbit: heterogenious in T1 weighted; hypersignal T2with a fluid level within the mass and enhancement of its internal septation following contrast administration

The mass was then carefully resected via a lateral orbitotomy and a drain was inserted into the remaining lesion. After surgery, the degree of proptosis was reduced and visual acuity was unimpaired. During 1 year of follow-up, hemorrhagic episodes have not recurred in this patient.

\section{DISCUSSION}

Lymphatic malformations, previously known as lymphangiomas, are benign vascular tumors usually diagnosed in early childhood and represent $1-3 \%$ of orbital masses [3]. Histologically, lymphatic malformations consist of enlarged, nonencapsulated channels lined with a single layer of endothelium [4].

The International Orbital Society characterizes orbital lesions by flow: type 1 (no flow), type 2 (venous flow), and type 3 (arterial flow) lesions. Orbital lymphangiomas are currently classified as type 1 (no flow) lesions, having minimal internal blood flow, with little to no connection with the vascular system [5].
Along with flow characteristics, orbital lymphangiomas are further classified as:

- $\quad$ superficial (presenting as a subcutaneous cyst);

- $\quad$ deep (orbital infiltration);

- combined (superficial and deep components);

- complex (intracranial or head and neck infiltration) [4].

There is no significant race or sex predilection, although lymphangiomas seem to be slightly more reported in females.

Orbital lymphangioma may produce a myriad of symptoms. Most patients present with symptoms in the first decade of life. The deeper lesions are classically going unrecognized until a hemorrhage into the lesion leads to proptosis [4, 6]. Patients also may present with a sudden increase in swelling or proptosis during an episode of upper respiratory tract infection [7]. Occasionally, patients present with gradual 
progression of proptosis and a restriction of ocular motility [4]. Other presentations can include globe displacement, physical disfigurement, mechanical blepharoptosis (which can obstruct visual development and result in amblyopia), and compressive optic neuropathy if left untreated after an acute hemorrhage into the lesion [4]. Early and effective treatment, especially in children, is therefore crucial to preserving vision and preventing amblyopia.

Orbital imaging is essential in helping make the correct diagnosis and identifying the extent of the lesion. CT and MRI typically demonstrate a cluster of non-enhancing "grape-like" cystic lesions with internal septations [8]. MRI can often delineate fluid-fluid levels within the cyst that represent layering of blood products from a recent intralesional bleed. Blood will appear hyperintense on T1 imaging. These unencapsulated lesions often demonstrate an infiltrative growth pattern, intertwining extensively with other orbital structures. Unlike a capillary hemangioma that is well encapsulated, lymphatic malformations frequently cross anatomic boundaries and tissue planes [8].

Ultrasound is another imaging modality that can aid in diagnosis. Typical characteristics of a lymphatic malformation on ultrasound include an irregular shape with poorly outlined borders, areas of high reflectivity, no change with Valsalva maneuver, and no vascular connection [9].

In terms of treatment, it is important to note that the lesions are generally unencapsulated and infiltrative. They can surround vital structures of the orbit, leading to the inability for complete excision by surgery without the risk of harm to these adjacent structures. Other methods of treatment, such as sclerotherapy, have therefore been used. However, these, too, have accompanying risks of increasing pressure in the orbit due to the volume of injecting fluid and resulting edema from inflammation due to the agents [10].

In conclusion, orbital lymphangioma should be considered in any case of proptosis in childhood. Imaging features are very helpful by showing an unencapsulated cystic mass with fluid levels. A multidisciplinary approach is needed, and the treatment has to be as conservative as possible if vision is not at risk and cosmesis is acceptable.

\section{REFERENCES}

1. Tunç M, Sadri E and Char DH. Orbital lymphangioma: an analysis of 26 patients. British Journal of Ophthalmology. 1999; 83(1), 76-80.

2. Char DH. Char DH. Clinical ocular oncology; 1997.

3. Hill III RH, Shiels WE, Foster JA, Czyz CN, Stacey A, Everman KR and Cahill KV. Percutaneous drainage and ablation as first line therapy for macrocystic and microcystic orbital lymphatic malformations. Ophthalmic Plastic \& Reconstructive Surgery. 2012; 28(2), 119-125.

4. Saha K and Leatherbarrow B. Orbital lymphangiomas: a review of management strategies. Current opinion in ophthalmology. 2012; 23(5), 433-438.

5. Harris GJ. Orbital vascular malformations: a consensus statement on terminology and its clinical implications. Orbital Society. American journal of ophthalmology. 1999; 127(4), 453-455.

6. Lally SE. Update on orbital lymphatic malformations. Current opinion in ophthalmology. 2016; 27(5), 413-415.

7. Kalisa P, Zieleghem BV, Roux $\mathrm{P}$ and Meire F. Orbital lymphangioma: clinical features and management. Bulletin de la Société belge d'ophtalmologie. 2001; 282, 59-70.

8. Khan SN and Sepahdari AR. Orbital masses: CT and MRI of common vascular lesions, benign tumors, and malignancies. Saudi Journal of Ophthalmology. 2012; 26(4), 373-383.

9. Harris GJ, Sakol PJ, Bonavolontà G and De Conciliis C. An analysis of thirty cases of orbital lymphangioma: pathophysiologic considerations and management recommendations. Ophthalmology. 1990; 97(12), 1583-1592.

10. Raichura ND, Alam MS, Noronha VO and Mukherjee B. A prospective study of the role of intralesional bleomycin in orbital lymphangioma. Journal of American Association for Pediatric Ophthalmology and Strabismus. 2017;21(2), 146151. 